\title{
Severe esophagitis in a patient with gastro- intestinal stromal tumor treated with imatinib
}

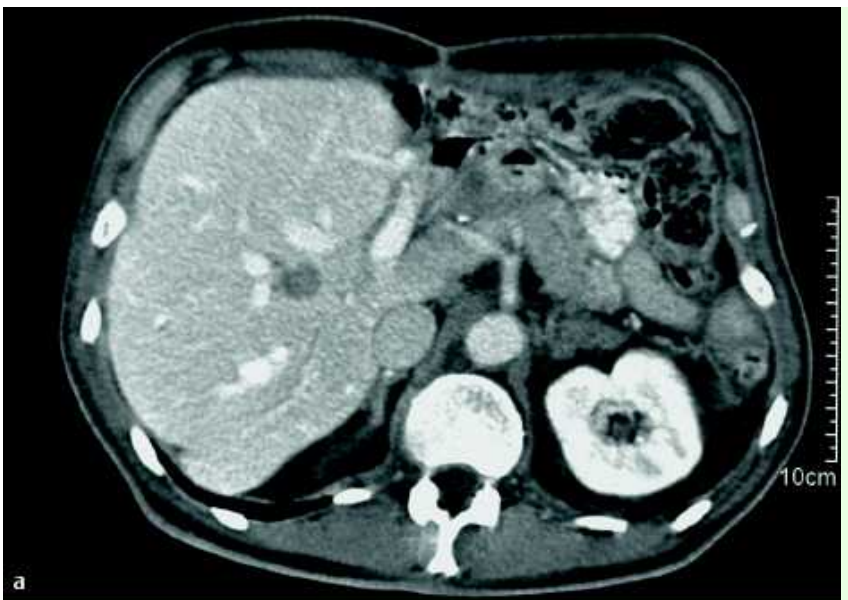

Fig. 1 a CT scan image before treatment with imatinib: multiple hepatic lesions in the right and left lobes. The biggest one measures $1.5 \mathrm{~cm}$. b CT scan image after treatment with imatinib: small hypodense lesions in the liver. The largest, in segment VIII, shows reduction of the intralesional enhancement and a diameter of $1.2 \mathrm{~cm}$.
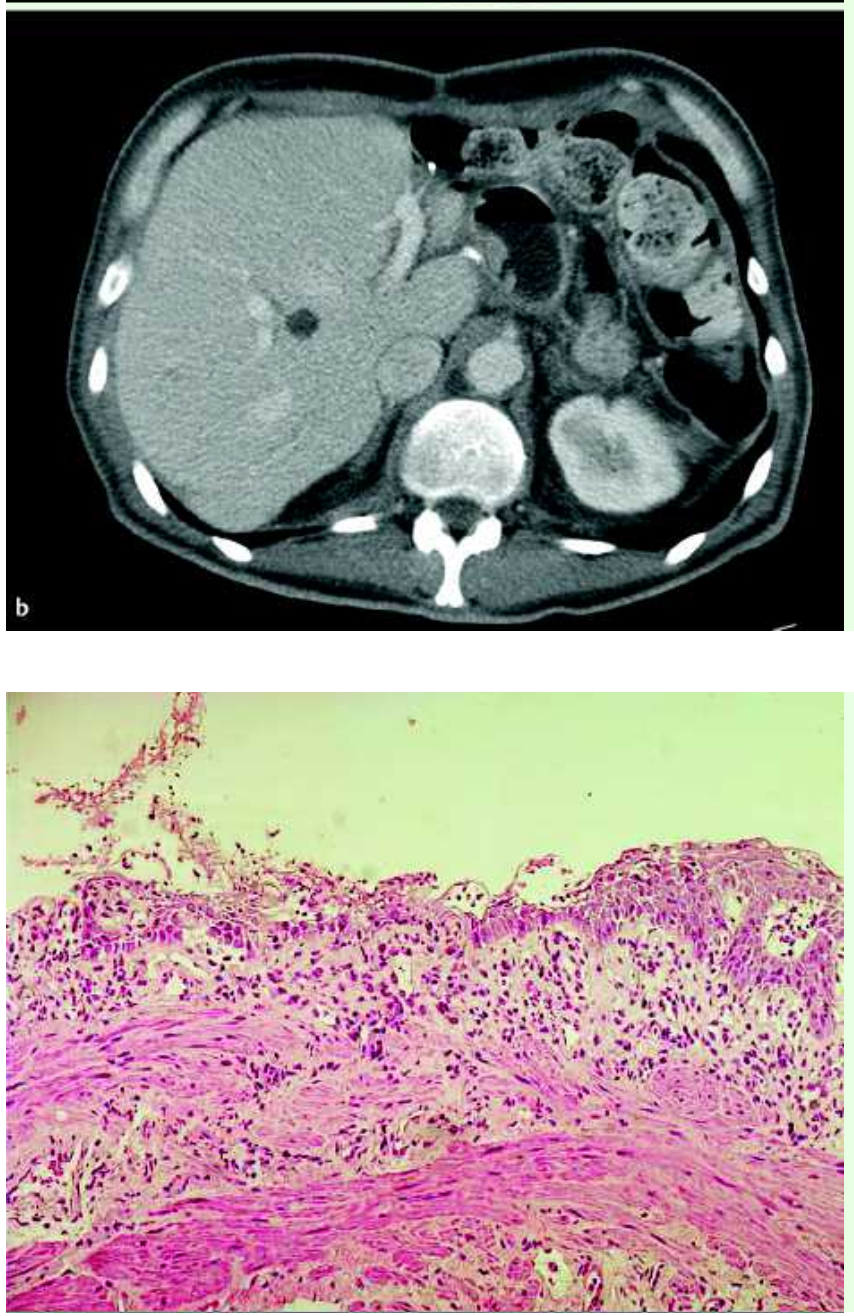

Fig. 3 Histological analysis of the esophageal mucosa indicates grade III esophagitis.

Imatinib is a specific inhibitor of tyrosine kinase and represents the standard treatment for metastatic and unresectable gastrointestinal stromal tumor (GIST) $[1,2]$. Imatinib is orally administered and

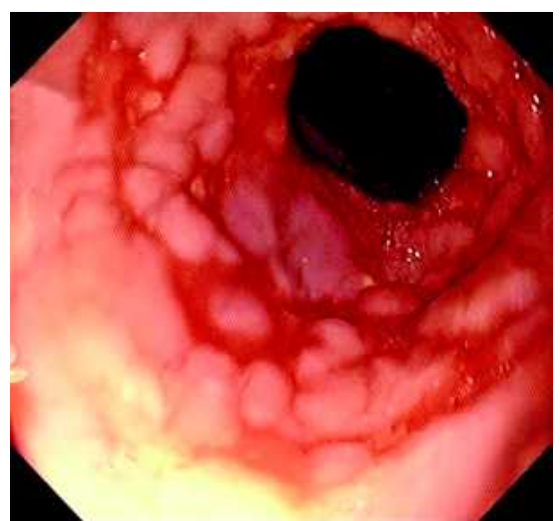

Fig. 2 Esophagogastroduodenoscopy image: severe erosive-ulcerative lesions of the esophageal mucosa.

A 56-year-old man with a high-risk GIST underwent gastric resection and liver metastasectomy. He was started on treatment with imatinib $400 \mathrm{mg} /$ day, which was prematurely suspended because of grade 3 dyspepsia. The patient restarted the treatment with imatinib when metastasis recurred in the liver and showed a good response ( $\bullet$ Fig. 1). However, during the treatment the patient reported severe dysphagia and retrosternal burning pain that was aggravated by food and water intake, and he experienced a weight loss of $11 \mathrm{~kg}$ in 2 months. Proton pump inhibitors, antacids, and prokinetics were ineffective. The only identifiable responsible agent was imatinib. We suggested splitting the drug intake into two administrations and performed esophagogastroduodenoscopy shortly afterwards. This showed erosive-ulcerative lesions of the esophageal mucosa starting at $27 \mathrm{~cm}$ from the dental arches and ending at the gastroesophageal anastomosis ( $\bullet$ Fig. 2). Some biopsies were taken and a diagnosis was made of grade III esophagitis with granulation tissue and necrotic material at the bottom of the ulcerative lesion ( Fig.3). Two months after treatment suspension, the patient's clinical condition is greatly improved.

In our patient, severe esophagitis occurred during treatment with imatinib. This case demonstrates that in clinical practice it is necessary to pay attention to every symptom reported by patients before irreversible damage appears: firstly, in or- 
der to avoid prolonged or definitive suspension of the drug, which limits the effect of therapy in responder patients, and, secondly, to avoid delay in beginning the second-line therapy $[4,5]$.

In conclusion, imatinib is generally well tolerated, but every unusual symptom needs to be regarded as suspicious and to be carefully investigated.

Endoscopy_UCTN_Code_CCL_1AB_2AC_3AB

Endoscopy_UCTN_Code_CCL_1AB_2AC_3AZ

M. Saponara ${ }^{1}$, M. Di Battista ${ }^{1}$, C. Lolli ${ }^{1}$, M. A. Pantaleo ${ }^{1}$, F. Azzaroli ${ }^{2}$, D. Santini ${ }^{3}$, V. Di Scioscio ${ }^{4}$, F. Catena ${ }^{5}$, M. Astorino ${ }^{1}$, A. Maleddu ${ }^{1}$, G. Biasco ${ }^{1}$

1 Department of Hematology and Oncology Sciences "L \& A Seràgnoli”, S. Orsola-Malpighi Hospital, University of Bologna, Italy

2 Department of Internal Medicine and Gastroenterology, S. Orsola-Malpighi Hospital, University of Bologna, Italy

3 Department of Pathology, S. OrsolaMalpighi Hospital, University of Bologna, Italy

4 Department of Radiology, S. OrsolaMalpighi Hospital, University of Bologna, Italy

${ }^{5}$ Emergency Surgery and Transplant Department, S. Orsola-Malpighi Hospital, University of Bologna, Italy

\section{References}

1 Demetri GD, von Mehren M, Blanke CD et al. Efficacy and safety of imatinib mesylate in advanced gastrointestinal stromal tumors. N Engl J Med 2002; 347: 472 - 480

2 Blanke CD, Demetri GD, von Mehren $M$ et al. Long-term results from a randomized phase II trial of standard- versus higher-dose imatinib mesylate for patients with unresectable or metastatic gastrointestinal stromal tumors expressing KIT. J Clin Oncol 2008; 26: 620-625

3 Van Glabbeke M, Verweij J, Casali PG et al. Predicting toxicities for patients with advanced gastrointestinal stromal tumours treated with imatinib: a study of the European Organisation for Research and Treatment of Cancer, the Italian Sarcoma Group, and the Australasian Gastro-Intestinal Trials Group (EORTC-ISG-AGITG). Eur J Cancer 2006; 42: 2277-2285

4 Blay JY, Le Cesne A, Ray-Coquard I et al. Prospective multicentric randomized phase III study of imatinib in patients with advanced gastrointestinal stromal tumors comparing interruption versus continuation of treatment beyond 1 year: the French Sarcoma Group. J Clin Oncol 2007; 25: 1107- 1113

5 Demetri GD, van Oosterom AT, Garrett CR et al. Efficacy and safety of sunitinib in patients with advanced gastrointestinal stromal tumour after failure of imatinib: a randomised controlled trial. Lancet 2006; 368: $1329-1338$
Bibliography

DOI 10.1055/s-0028-1119457

Endoscopy 2009; 41: E67-E68

(c) Georg Thieme Verlag KG Stuttgart - New York · ISSN 0013-726X

Corresponding author

\section{Saponara, MD}

Department of Hematology and

Oncology Sciences “L \& A Seràgnoli”

University of Bologna

S. Orsola-Malpighi Hospital

via Massarenti 9

40138 Bologna

Italy

Fax: +39-051-6364037

maristellasaponara@yahoo.it 